\title{
Collaborative Trust-Based Shortest Secure Path Discovery in Mobile Ad Hoc Networks ${ }^{\star}$
}

\author{
Seungtak Oh, Chilgee Lee, and Hyunseung Choo \\ School of Information and Communication Engineering, \\ Sungkyunkwan University, \\ 440-746, Suwon, Korea \\ +82-31-290-7145 \\ choo@ece.skku.ac.kr
}

\begin{abstract}
As routes in MANET are composed of only mobile terminals based on a multi-hop mechanism, MANET may encounter severe situations where intermediate nodes disturb delivering packets adversely. Accordingly, the stability of routing protocols is very important for operating the ad hoc networks efficiently and securely. However finding a secure end-to-end path is not easy, since the nodes in MANET are characterized by uncertain trust relationship each other. In this paper, a comprehensive mechanism for discovering the most secure and shortest paths, is proposed. This proposed mechanism is based on the Dijkstra algorithm, and regards distance weight and trust weight highly. The metric value which can determine the routing path is to be extracted from the distance value and the trust level. According to the simulation results, even though the communication cost increases up to about $8 \%$, the security level of the shortest path is improved significantly.
\end{abstract}

\section{Introduction}

Mobile Ad Hoc Network (MANET) is an infrastructureless network, consisting of a set of mobile nodes without any support of base stations or access points. A node in MANET is responsible for relaying packets to other nodes in addition to a terminal station. Then some packets transmitted from a source node can be delivered to a destination node by way of various intermediate nodes, under the store-and-forward function. Therefore, MANET can be easily applied to next generation applications such as telematics, USN and home networks, because of its flexibility and agility in establishment.

Unfortunately, in the presence of malicious nodes, MANET is highly vulnerable to attacks, due to dynamically changing topology, frequent appearance of nodes, and lack of centralized security infrastructure. An attacker can attempt to cause legitimate packets to be routed in dysfunctional ways or inject packets

\footnotetext{
* This research was supported by the Ministry of Information and Communication, Korea, under the Information Technology Research Center support program supervised by the Institute of Information Technology Assessment, IITA-2005-(C10900501-0019). Corresponding author: H. Choo.
} 
into the network by an attack to consume valuable network resources. In the case of wormhole attack that is not easy to detect, an attacker receives packets in one part of the network and tunnels them to another part and replays them in the different part. Consequently, it is very important to realize robust and secure routing protocols.

There are basically two stems to solve these problems in MANET: the one is how to identify legitimate users, the other is how to establish secure paths. Many researches are currently interested in the user identification using certificates [4, 6, ,7, but it is not easy to realize without any certificate authority(CA) infrastructure. And also several secure routing protocols have been developed so far, but it is very hard to find out the best one among these protocols because of its preliminary conditions such as sharing a secret key, requiring a certain infrastructure, and so on.

With regard to the insecurity of MANET environment, a further study on combining user identification and secure routing protocol will be intensely needed. By now, there have been some works on trust relationship so as to enhance the security level of the routing paths, but has not been any research upon how to initialize the trust level on each node and estimate the trust level of other nodes. In this paper, a solution is presented, discovering the shortest and most secure path by employing a new collaborative trust relationship mechanism. This mechanism considers the distance weight and the trust weight, and shows how to assign the initial trust weight and reckon the trust level. In simulation, it is confirmed that the proposed mechanism increases the communication cost up to about $8 \%$, but the security level of the shortest path is improved significantly.

The remainder of this paper is organized as follows. The related works regarding secure routing protocols in MANET are presented in Section 2. In section 3 , the solution is explained in detail. In Section 4, the results of simulation are discussed, and the effectiveness of the proposed mechanism is verified. Finally, Section 5 concludes this paper.

\section{Related Works}

Current efforts toward the design of a secure MANET environment have mainly focused on user identification by means of a certificate and representative secure routing protocols. SRP [1] relies on the availability of a $\operatorname{Security} \operatorname{Association}(S A)$, a shared key $K_{S T}$, between the source node $(S)$ and the destination node $(T)$, without intermediate nodes requiring cryptographic validation of control traffic. The success of this protocol depends on an efficient distribution of shared secret keys. S. Capkun and J. Hubaux introduce a realistic solution that enables the establishment of secret keys between all pairs of nodes through offline authority or fully self-organized mechanism 2]. This solution, however, has a potential risk to share a secret key with a malicious node, as the indirect user identification between a pair of nodes fully relies on the security association of its friend node. Another secure routing protocol, ARAN 3] requires the use of a trusted certificate server, and is composed of two distinct stages. The goal of the first stage is to 
enable the source node to verify whether the intended destination is reached or not. The second stage is primarily used for secure discovery of the shortest path. ARAN introduces authentication, message integrity and non-repudiation, but is not a suitable mechanism for an isolated MANET environment, due to the requirement for the certificate server. And SEAD routing protocol is proposed in [5]. It makes use of efficient one-way hash chains. The basic idea of SEAD is to authenticate the sequence number and the metric of a routing table update message using hash chains elements. An attacker cannot generate any value, in the hash chain of the node, which the node will use in the future as it is impossible to invert a one-way hash function. SEAD, however, cannot prevent the same distance attack where a node receives an advertisement for a particular sequence number and metric and then it readvertises the same sequence number and metric.

On the other hand, N. Pissinou, T. Ghosh and K. Makki introduce an extension of the AODV 8$]$ routing protocol which is called T-AODV $[9,10]$. When a node desires finding a path to another node, it initiates route discovery by broadcasting a Route Request packet(RREQ). The packet header contains a trust level field, in addition to the other fields in AODV RREQ. When an intermediate node receives the RREQ packet, it rebroadcasts the packet, after modifying the trust level field to include the trust level of the node transmitting it the RREQ. Every node verifies the rebroadcasted RREQ packet from its next node to verify transmitting of proper information. If the validation fails, it immediately broadcasts a warning message questioning the trustworthiness of that specific node. The final route selection is based upon the trust level metric. However, this is not sufficient in figuring out how to share the trust level, and calculate the trust levels set on each of the edges of a route path.

In 11], A. Pirzada and his group address a trust-based routing scheme. Whenever a node transmits data, it places its receiver in promiscuous mode. As soon as it hears its intermediate neighbor forwarding the packet, the sender node checks the integrity of the packet by verification process. If the integrity check passes, it confirms that the intermediate node has acted in a good manner and so its direct trust counter is increased. Similarly, if the integrity check fails or the intermediate node does not transmit the packet, its corresponding direct trust counter is decreased. In order to propagate trust information, each node appends the direct trust value of the preceding node from which it had received the RREQ packet before forwarding the packet. This mechanism can detect grey hole, black hole, and modification attacks, but has a critical flaw that most of nodes must always set its receiver in promiscuous mode, and then the information of other nodes can be easily disclosed.

\section{Shortest Secure Path Discovery Mechanism}

In Figure1, simply applying the Dijkstra algorithm, which can search the shortest path, may undergo a serious problem such as wormhole attacked covertly by a malicious node $\mathrm{M}$ in MANET. 


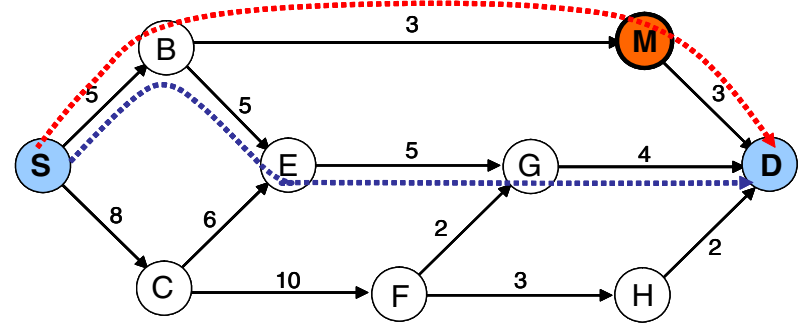

Fig. 1. Single-Source Shortest Paths

If the trust level is considered, the shortest path is established by following $\mathrm{S} \rightarrow \mathrm{B} \rightarrow \mathrm{E} \rightarrow \mathrm{G} \rightarrow \mathrm{D}$, but if not, it is constructed by following $\mathrm{S} \rightarrow \mathrm{B} \rightarrow \mathrm{M}$ $\rightarrow$ D. Eventually, the shortest path which is composed of a malicious node can't afford a secure path absolutely. To overcome this problem, we propose a new mechanism, which can find an secure path by appending the trust weight to every edge among nodes, in addition to the distance weight. A graph $G(V, E)$ represents a set of nodes and a set of edges in MANET respectively. Here a node means an element in $\mathrm{V}$ and an edge means a connection between two nodes containing the distance weight and the trust weight. Then the two edge elements are handled respectively. The running condition of the proposed mechanism requires that secure routing protocols, such as T-AODV, discovering the shortest path ought to have the trust weight information regarding the previous nodes in response packets.

\subsection{Trust Level}

The edge element $E$ may demonstrate the trust weight between a pair of nodes, and has a real number between 0 and 1 . When a node attempts to connect to MANET in the proposed mechanism, initially, certificates have to be exchanged with each other through a point-to-point network, that is, secure side channel, following realistic mutual identification with neighbor nodes.

Otherwise, connection to MANET is impossible. Mutual authentication by certificates is not explained further in this paper.

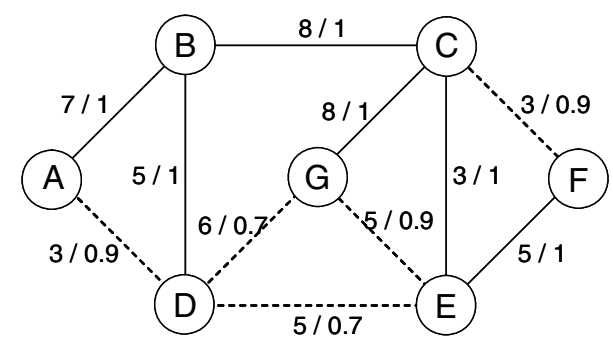

Fig. 2. Edges with Distance and Trust Weight 
In Figure 2, all edges have two numbers that the first number means the distance weight and the second number the trust weight. And the solid line edges represent the situation that two nodes, facing each other, execute the mutual authentication directly, its trust weight is always 1 . Otherwise, the dotted line edges mean that no mutual authentication occurs directly. Here Trust $(P)$, the trust level from a source node to a destination node, can be evaluated using the following equation.

$$
\operatorname{Trust}(P)= \begin{cases}t w, & \text { for } \eta=1 \\ \frac{\min _{i=0}^{p}\left(t w_{i}\right)}{\ln (\eta+1)}, & \text { for } \eta>1\end{cases}
$$

Here, $\eta$ represents for the number of hops. The trust level is always 1 on the condition that mutual authentications are executed offline and the number of hops is 1. Otherwise, the trust level depends on the minimum of the trust weights from a source node to a destination node and the number of hops. In Equation (1), the natural $\log , \ln (\eta+1)$, is used when the number of hops is greater than 1, so that the trust level can be differentiated by the number of hops even though the same trust level. That is, the more the number of hops increases, the less the trust level decreases.

Furthermore, if several routing paths to a destination node are discovered, the path having the highest trust level is selected as the shortest secure path.

\subsection{Routing Path Discovering Algorithm}

In addition to Trust $(P)$, it is essential to aggregate all the distance weights of a path to find the shortest path. Distance $(P)$, the distance value from a source node to a destination node, can be defined as Equation (2).

$$
\begin{aligned}
\operatorname{Distance}(P) & =\sum_{i=1}^{p} d w\left(v_{i-1}, v_{i}\right) \\
\operatorname{Metric}(P) & =\frac{\operatorname{Distance}(P)}{\operatorname{Trust}(P)}
\end{aligned}
$$

That is, Distance $(P)$ aggregates the distance weight $(d w)$ from a source node to a destination node. The shortest routing path may be the path with the smallest distance value to the destination node. In addition to the distance, the proposed algorithm manages the Trust $(P)$ and its weight. Trust $(P)$ basically chooses the smallest trust weight of all the edges on a path. Eventually a new $\operatorname{Metric}(P)$, which directs the shortest and secure routing path in Equation (3), is defined.

In Table 1, the proposed algorithm, regarding the critical functions, is described. The proposed algorithm uses the metric value in order to determine the most efficient path. 
Table 1. Proposed Algorithm

\begin{tabular}{|c|c|}
\hline$\overline{\operatorname{Proposed}(G, w, s)}$ & $\operatorname{Relax}(u, v, d w, t w)$ \\
\hline 1. Initialize-Single-Source $(G, s)$ & 1. if $\left(\mathrm{m}[u]+\left(\mathrm{dw}(u, v) / \mathrm{tw}_{\mathrm{w}}(u, v)\right)\right)<\mathrm{m}[v]$ \\
\hline 2. $\mathrm{d}[s] \leftarrow 0$ & 2. do $\mathrm{d}[v] \subseteq \mathrm{d}[u]+\mathrm{dw}(u, v)$ \\
\hline 3. $t[s] \leftarrow 1$ & $\mathrm{t}[v] \subseteq \min \left(\mathrm{t}[u], \mathrm{tw}_{\mathrm{w}}(u, v)\right)$ \\
\hline 4. $\mathrm{m}[s] \leftarrow 0$ & $/ \log (\mathrm{h}[v]+1)$ \\
\hline 5. $\operatorname{pred}[s] \leftarrow N I L$ & 4. $\quad \mathrm{m}[v] \subseteq \mathrm{d}[v] / \mathrm{t}[v]$ \\
\hline 6. $Q \leftarrow \mathrm{V}[G]$ & Decrease-Key $(\mathrm{Q}, \mathrm{v}, \mathrm{m}[v])$ \\
\hline 7. while Non-Empty $(Q)$ & $\operatorname{pred}[v] \subseteq u$ \\
\hline 8. do $u \leftarrow$ Extract-Min $(Q)$ & \\
\hline 9. $\quad \mathrm{S} \leftarrow \mathrm{S} \cup\{u\}$ & \\
\hline $\begin{array}{l}\text { 10. for each } v \subseteq \operatorname{Adj}[u] \\
\text { 11. } \quad \text { do } \operatorname{Relax}(u, v, d w, t w)\end{array}$ & \\
\hline
\end{tabular}

\section{Performance Evaluation}

The Dijkstra algorithm written in $\mathrm{C}++$ language is used for simulation, and some functions are appended or modified to realize the proposed scheme. The modified program is executed a total of five times under such conditions, as the network consists of 130 nodes and 120 links. More detailed simulation environments are presented in Table 2. The distance weight is randomly generated from a minimum of 3 to a maximum of 52, and the trust weight from a minimum of 0.3 to a maximum of 1 .

Table 2. Simulation Environments

\begin{tabular}{cccr}
\hline Times & Vertices & Edges & Paths \\
\hline 1st & 130 & 503 & 124 \\
2nd & 130 & 501 & 123 \\
3rd & 130 & 500 & 126 \\
4th & 130 & 503 & 127 \\
5 th & 130 & 503 & 128 \\
\hline Total & 650 & 2,510 & 628 \\
\hline
\end{tabular}

Figure 3 presents the characteristic of the number of paths in terms of the number of hops, which composes some of the simulation results. In Figure 3, we can find the phenomenon that the distribution curve of hops highly increases at approximately 4 hops of both algorithms, which have 628 paths equally, and that there is no path consisting of 8 hops or more, in the proposed algorithm. This is the reason that the proposed algorithm intends to discover the most secure path within a nearby boundary, because the more the number of hops increases, the less the trust level decreases.

Figure 4 shows the fact that distance average in the Gaussian distribution generally increases from 128.66 to 136.82 , and then, compared with Dijkstra, the distance graph is shifted slightly toward the right. Therefore, it is expected that 


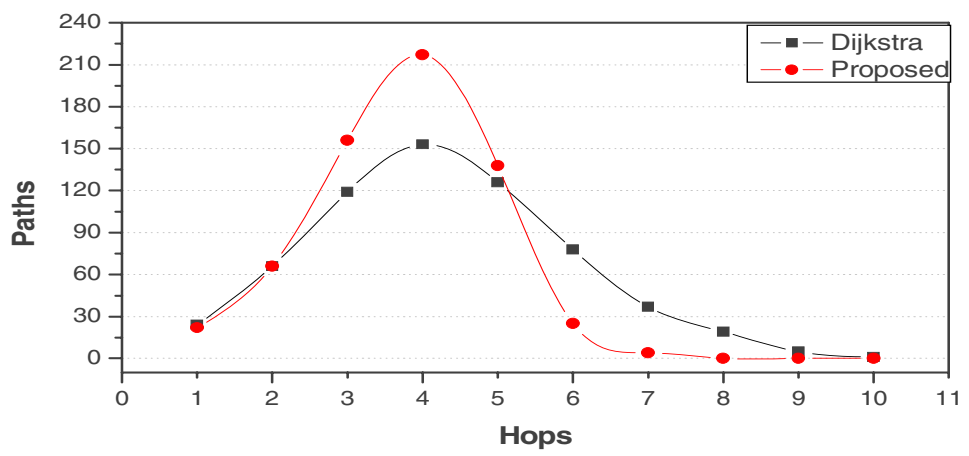

Fig. 3. No. of Paths in terms of the No. of Hops

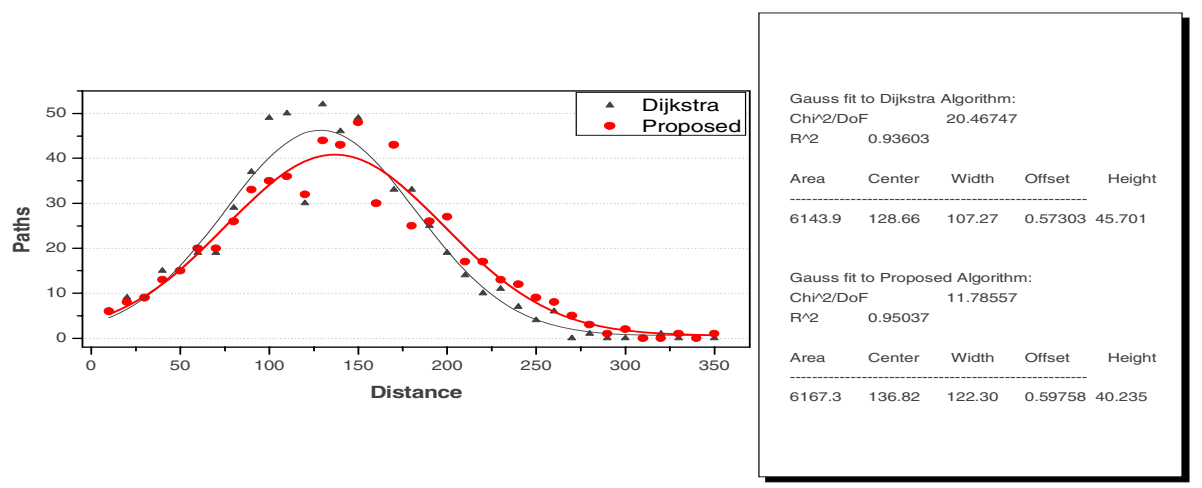

Fig. 4. Correlation of Distance and Paths

the increase in distance will bring the communication cost up to about $8 \%$. However, it is possible to obtain more secure paths by adjusting the threshold level appropriately. For instance, if the threshold level is set to 0.4 , only the path which its trust level is more than the threshold level is to be accepted as a secure one.

\section{Conclusion}

The proposed mechanism is located in the sublayer of secure routing protocols considering the trust level. In offering routing information from them, it is possible to select the most secure and shortest path from the unreliable MANET environment, by means of the ability to perceive the trust levels of opposite nodes. Even though the proposed mechanism works with the trust weight as well as the distance weight, it does not make any differences in a viewpoint of the number of hops, except for rising the communication cost slightly. Consequently, discovering the shortest secure paths in MANET by using the proposed mechanism, provides one of the best solutions, and basically prevents internal malicious nodes from attacking or disguising, such as in the case of a wormhole 4. 


\section{References}

1. P. Papadimitratos, Z.J. Haas, "Secure routing for mobile ad hoc networks", SCS Communication Networks and Distributed Systems Modeling and Simulation Conference(CNDS 2002), 2002.

2. Srdjan Capkun, Jean-Pierre Hubaux and Levente Buttyan, "Mobility Helps Peerto-Peer Security", IEEE Transactions on Mobile Computing, VOL. 5, NO. 1, pp. 43-51, 2006.

3. K. Sanzgiri, B.Dahill, B.N. Levine, C. Shields, E.M. Belding-Royer, "A Secure Routing Protocol for Ad Hoc Networks", Proceedings of the 10 th IEEE International Conference on Network Protocols (ICNP02), 2002.

4. Karlof, David Wagner, "Secure routing in wireless sensor networks: attacks and countermeasures", Ad Hoc Networks 1, pp. 293-315, 2003.

5. Y.C. Hu, D.B. Johnson, A. Perrig, "SEAD: secure efficient distance vector routing for mobile wireless ad hoc networks", Proceedings of the 4th IEEE Workshop on Mobile Computing Systems and Applications(WMCSA 2002), pp. 3-13, 2002.

6. G. Montenegro and C. Castelluccia. "Statistically Unique and Cryptographically Verifiable(SUCV) Identifiers and Addresses", Proc. Symp. Networks and Distributed Systems Security(NDSS 2002), Internet Society, pp. 87-99, 2002.

7. Srdjan Capkun, Levente Buttyan, Jean-Pierre Hubaux, "Self-Organized PublicKey Management for Mobile Ad Hoc Networks", IEEE Transactions on Mobile Computing, VOL. 2, NO. 1, pp. 52-64, 2003.

8. C.E. Perkins, E.M. Royer, "Ad-hoc on-demand distance vector(AODV) routing", in: The Second IEEE Workshop on Mobile Computing Systems and Applications, New Orleans, LA, USA, February 1999.

9. Niki Pissinou, Tirthankar Ghosh, Kia Makki, "Collaborative Trust-Based Secure Routing in Multihop Ad Hoc Networks", Proceedings of Networking '04:Springer Verlag, Series:Lecture Notes in Computer Science, Vol.3042, pp. 1446-1451, Athens, Greece, May 9-14, 2004.

10. Tirthankar Ghosh, Niki Pissinou, Kia Makki, "Collaborative Trust-based Secure Routing Against Colluding Malicious Nodes in Multi-hop Ad Hoc Networks", Local Computer Networks, 2004. 29th Annual IEEE International Conference on, pp. 224-231, Nov 16-18, 2004.

11. Asad Amir Pirzada, Amitava Datta, Chris McDonald, "Trust-based routing for ad-hoc wireless networks", Networks, 2004.(ICON 2004). Proceedings. 12th IEEE International Conference on Volumn 1, pp. 326-330, Nov 16-19, 2004. 